\title{
Duration of Postoperative Fever as a Simple and Useful Prognostic Indicator in Gastric Cancer Patients
}

\author{
Hirohiko Kuroda,* Hiroaki Saito,$\dagger$ Yuki Murakami, $\$$ Yuji Shishido, $\$$ Kozo Miyatani, $\$$ Tomoyuki Matsunaga $\$$ and \\ Yoshiyuki Fujiwarat \\ *Department of Surgery, Japanese Red Cross Masuda Hospital, Masuda 698-8501, Japan, †Department of Surgery, Japanese Red \\ Cross Tottori Hospital, Tottori 680-8517, Japan, and $\neq$ Division of Gastrointestinal and Pediatric Surgery, Department of Surgery, \\ School of Medicine, Faculty of Medicine, Tottori University, Yonago 683-8504, Japan
}

\begin{abstract}
Background Postoperative inflammation is associated with cancer progression in several cancers. However, the prognostic significance of postoperative fever remains unclear in gastric cancer patients.
\end{abstract}

Methods We enrolled 442 patients with a histopathological diagnosis of gastric adenocarcinoma who underwent curative surgery.

Results The mean duration of postoperative fever $\geq$ $37^{\circ} \mathrm{C}$ was 8.7 days (range: $0-186$ days) and significantly longer in patients with advanced gastric cancer, venous invasion, and open or total gastrectomy vs. patients with early gastric cancer $(P=0.0072)$, no venous invasion $(P=0.025)$, laparoscopic gastrectomy $(P=0.027)$, and either proximal or distal partial gastrectomy $(P=0.0015)$. Five-year overall survival rates were $69.5 \%$ vs. $83.6 \%$ in the prolonged postoperative fever group ( $\geq 6$ days of $\geq$ $\left.37^{\circ} \mathrm{C}\right)$ vs. the nonprolonged group $\left(<6\right.$ days of $\left.\geq 37^{\circ} \mathrm{C}\right)$, respectively $(P=0.0008)$. In patients without ClavienDindo classification postoperative infectious complications grade $\geq 2$, 5 -year overall survival was $69.7 \%$ vs. $84.0 \%$ in patients with prolonged postoperative fever vs. those without, respectively $(P=0.0067)$. Five-year disease-specific survival was $85.9 \%$ vs. $93.1 \%$ in patients with prolonged fever vs. those without, respectively $(P=$ $0.041)$. Multivariate analysis indicated that postoperative fever was an independent prognostic indicator.

Conclusion Postoperative fever $\geq 37^{\circ} \mathrm{C}$ duration may be useful in predicting prognosis in gastric cancer patients.

Key words fever; gastric cancer; prognosis; recurrence

Corresponding author: Hiroaki Saito, MD, PhD

sai10@tottori-med.jrc.or.jp

Received 2021 March 17

Accepted 2021 July 7

Online published 2021 August 6

Abbreviations: AUC, area under the curve; CRP, C-reactive protein; DSS, disease specific survival; GC, gastric cancer; NSAIDs, non-steroidal anti-inflammatory drugs; OS, overall survival; ROC, receiver operating characteristic
Gastric cancer is one of the most common malignancies. Although recent progress in chemotherapy for gastric cancer has improved the prognosis for unresectable advanced and recurrent gastric cancer patients, it ranks third among all cancer-related deaths worldwide. ${ }^{1}$ Therefore, more progress in diagnosis and treatment is required to improve the prognosis for patients with gastric cancer.

Gastrectomy with regional lymph node dissection is the mainstay of gastric cancer treatment to achieve cure. However, many patients with gastric cancer experience recurrence even after complete tumor removal (R0 resection). Early detection of recurrence may contribute to survival after curative gastrectomy for gastric cancer. ${ }^{2}$ Recurrence occurs because of the existence of micrometastases that cannot be detected by standard diagnostics, including ultrasonography, computed tomography, and positron emission tomography. Therefore, it is extremely important to select gastric cancer patients with a high possibility of recurrence after curative gastrectomy and to perform extensive follow-up to improve patients' prognosis. To this end, it is indispensable to have useful prognostic indicators.

Accumulating evidence indicates that cancer patient's inflammation status is associated with prognosis. Postoperative fever is a host response to inflammation induced by surgery; therefore, it is likely that evaluating postoperative fever helps predict patients' prognosis. A small number of studies have investigated the prognostic value of postoperative fever in breast cancer [5, 6] and colorectal cancer [7-9]; however, the prognostic significance of postoperative fever remains unclear in gastric cancer patients. In this study, we aimed to investigate the prognostic value of postoperative fever in gastric cancer patients after radical gastrectomy.

\section{MATERIALS AND METHODS Patients}

This study involved a retrospective analysis of 442 gastric adenocarcinoma patients who underwent curative gastrectomy (R0 resection) at our institution between January 2001 and April 2013. Clinicopathological 
findings were determined according to the Japanese Classification of Gastric Carcinoma. ${ }^{3}$ All patients underwent either distal partial, proximal partial, or total gastrectomy with regional lymph node dissection. Fourteen patients received neoadjuvant chemotherapy, and 67 patients received adjuvant chemotherapy. No patients underwent radiation therapy. In principle, early GC patients visit our outpatient clinic every 6 months for the first 2 years and every 12 months for 3 years following the operation. They take blood test including tumor markers (carcinoembryonic antigen and carbohydrate antigen 19-9) every time and esophagogastroduodenoscopy and computed tomography once every 12 months. Advanced GC patients visit our outpatient clinic every 3 months for the first 2 years and every 6 months for 3 years following the operation. They also take a blood test including tumor markers every time and esophagogastroduodenoscopy and computed tomography once every 6 months. Causes of death and patterns of recurrence were determined by reviewing medical records, including laboratory data, ultrasonography, computed tomography, scintigrams, peritoneal punctures, and laparotomies, or by direct inquiry of family members. Body temperature was measured with an axillary thermometer at least four times a day after surgery, and the highest one was used in the analysis. In principal, an antipyretic was given with fever $\geq 38^{\circ} \mathrm{C}$. Serum albumin (Alb) concentration and total neutrophil count (NC), platelet count (PC), and lymphocyte count (LC) of the peripheral blood were measured within the month before surgery. The PNI was calculated as follows: $10 \times$ Alb concentration + $0.005 \times$ total LC. Neutrophil to lymphocyte ratio (NLR) and platelet to lymphocyte ratio (PLR) were calculated by dividing the $\mathrm{NC}$ and $\mathrm{PC}$ by $\mathrm{LC}$, respectively. The Institutional Review Board of our institution approved this study (17A147), and the need for informed consent was waived.

\section{Statistical analysis}

We evaluated differences between the two groups using the Mann-Whitney $U$-test. Youden's index was calculated using receiver operating characteristic (ROC) analysis to determine optimal cutoffs for postoperative fever duration in the survival analysis. Survival curves were calculated according to the Kaplan-Meier method. Differences between the curves were identified using the log-rank test. We performed multivariate analysis of factors considered prognostic for overall survival with Cox's proportional hazards model and a stepwise procedure. The covariates included in multivariate analysis were age, sex, tumor size, histology, depth of invasion, lymph node metastasis, lymphatic involvement, venous involvement, approach, lymph node dissection, type of gastrectomy, postoperative fever $\geq 37^{\circ} \mathrm{C}$ duration, NLR, and PLR. A $P$-value $<0.05$ was considered statistically significant. We used GraphPad Prism (GraphPad Software, Inc., La Jolla, CA) and Stat View (Abacus Concepts, Inc., Berkeley, CA) software for the statistical analyses.

\section{RESULTS}

We first determined the prognostic significance of postoperative fever duration and maximum postoperative temperature. We performed a ROC analysis for overall survival and compared the area under the curve (AUC) values to assess the discriminatory ability of postoperative fever duration and maximum postoperative temperature. The AUC of postoperative fever $\geq$ $37^{\circ} \mathrm{C}$ duration was $0.6168(P=0.00016,95 \%$ confidence interval: 0.5569-0.6766; Fig. 1a), which was higher than the AUC of postoperative fever $\geq 37.5^{\circ} \mathrm{C}$ duration (Fig. 1b), postoperative fever $\geq 38.0^{\circ} \mathrm{C}$ duration (Fig. 1c), or maximum postoperative temperature (Fig. 1d), indicating that postoperative fever $\geq 37^{\circ} \mathrm{C}$ duration was more useful than the other indicators to predict prognosis for gastric cancer patients.

Figure 2 shows the histogram of postoperative fever duration. The mean postoperative fever $\geq 37^{\circ} \mathrm{C}$ duration was 8.7 days (range: $0-186$ days). Table 1 shows the correlation between postoperative fever $\geq 37^{\circ} \mathrm{C}$ duration and patients' clinicopathological characteristics. Postoperative fever $\geq 37^{\circ} \mathrm{C}$ duration was significantly longer in patients with advanced gastric cancer, venous invasion, and open or total gastrectomy compared with those with early gastric cancer $(P=0.0072)$, no venous invasion $(P=0.025)$, laparoscopic gastrectomy $(P=$ $0.027)$, and either proximal or distal partial gastrectomy $(P=0.0015)$. We then determined the correlation between the postoperative fever $\geq 37^{\circ} \mathrm{C}$ duration and some prognostic indicators, including NLR, PLR, and PNI. The NLR were $2.6 \pm 15$ and $2.4 \pm 1$.5in patients with prolonged fever and those without prolonged fever, respectively $(P=0.1)$. The PLR were $171.9 \pm 85.7$ and $154.8 \pm 81.5$ in patients with prolonged fever and those without prolonged fever, respectively, and the difference was statistically significant $(P=0.024)$. The PNI was significantly lower in patients with prolonged fever $(47.2$ $\pm 6.0)$ than those without prolonged fever $(49.2 \pm 6.1 ; P$ $=0.0001$ ).

We next determined the correlation between postoperative fever $\geq 37^{\circ} \mathrm{C}$ duration and prognosis in gastric cancer patients. ROC analysis revealed an optimal cutoff value of postoperative fever $\geq 37^{\circ} \mathrm{C}$ duration for 
(a)

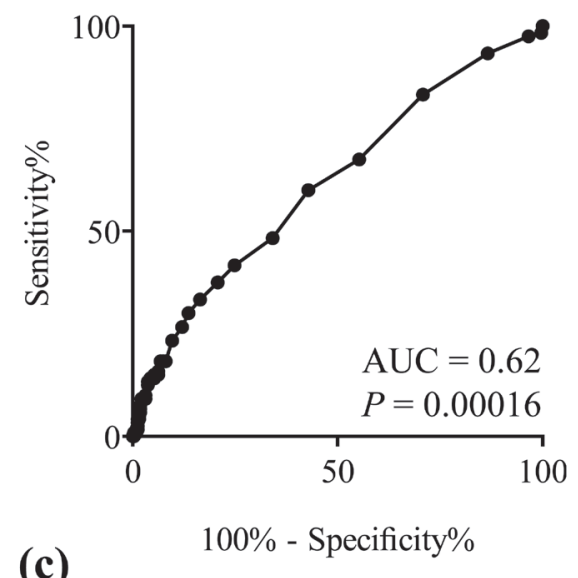

(c)

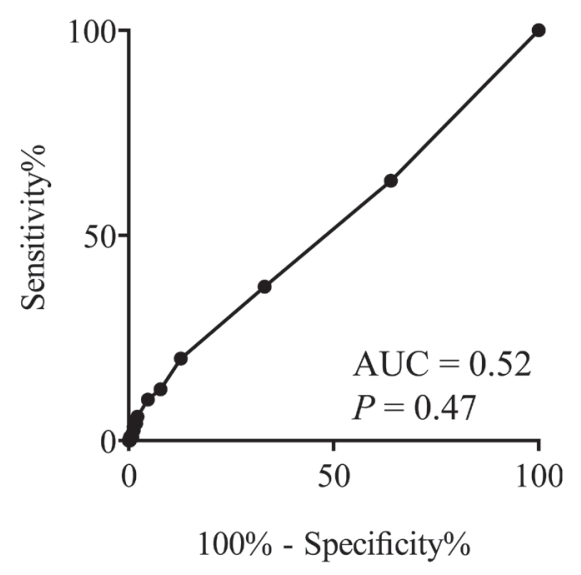

(b)
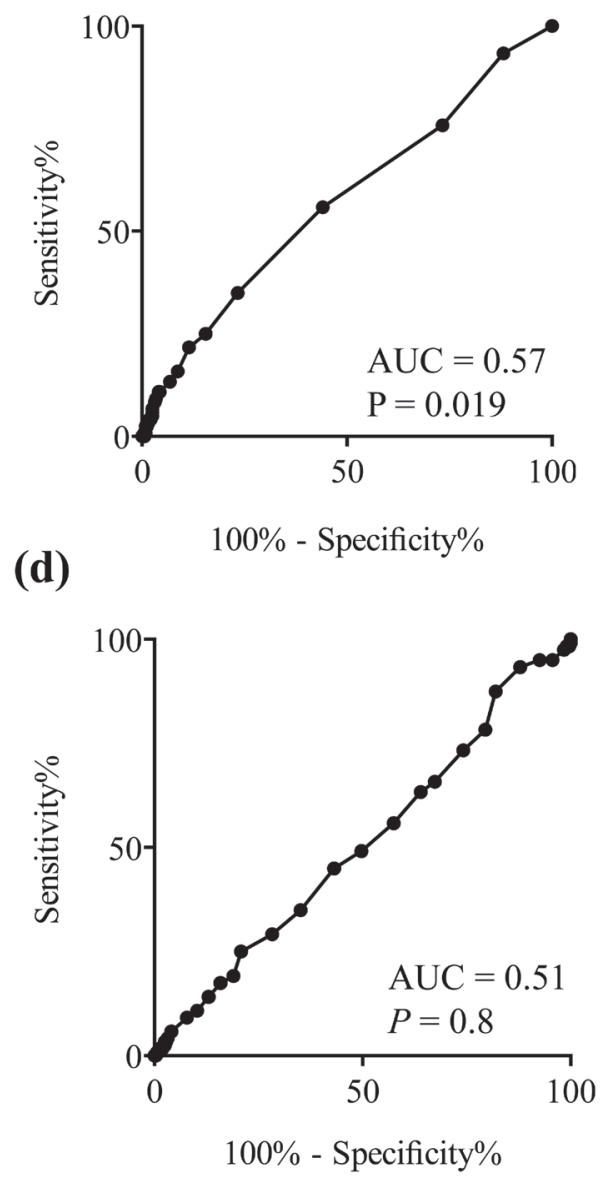

Fig. 1. Comparison of the areas under the receiver operating curves for outcome prediction (a) Duration of postoperative fever $\geq 37^{\circ} \mathrm{C}$, (b) duration of postoperative fever $\geq 37.5^{\circ} \mathrm{C}$, (c) duration of postoperative fever $\geq 38^{\circ} \mathrm{C}$, and $(\mathbf{d})$ maximum postoperative fever values.

overall survival of 5.5 (AUC $=0.6168, P=0.00016$ ). Based on these results, we divided patients into the following groups: prolonged postoperative fever duration group ( $\geq 6$ days at $\geq 37^{\circ} \mathrm{C}, n=210$ ) and a nonprolonged postoperative fever group ( $\leq 5$ days at $\geq 37^{\circ} \mathrm{C}, n=232$ ). The usages of anti-inflammatory analgesic, such as non-steroidal anti-inflammatory drugs (NSAIDs) or acetaminophen, after operation were $6.1 \pm 9.5$ and $3.4 \pm$ 5.0 in prolonged postoperative fever duration group and nonprolonged postoperative fever group, respectively, and the difference was statistically significant $(P=$ $0.0004)$. The postoperative fever $\geq 38^{\circ} \mathrm{C}$ durations was $0.72 \pm 0.83$ days vs. $2.3 \pm 4.1$ days in nonprolonged postoperative fever group vs. prolonged postoperative fever group, respectively $(P<0.0001)$. Furthermore, maximum postoperative temperature was $38.0 \pm$ $0.56^{\circ} \mathrm{C}$ vs. $38.4 \pm 0.63^{\circ} \mathrm{C}$ in nonprolonged postoperative fever group vs. prolonged postoperative fever group, respectively $(P<0.0001)$.

Five-year overall survival (OS) rates were $69.5 \%$ vs. $83.6 \%$ (prolonged postoperative group vs. nonprolonged postoperative fever group, respectively) and the difference was statistically significant $(P=0.0008$, Fig. 3a). Five-year disease specific survival (DSS) rates were $85.7 \%$ vs. $92.1 \%$ (prolonged postoperative group vs. nonprolonged postoperative fever group, respectively) and the difference was statistically significant $(P=0.032$, Fig. 3b). With regard to the correlation between prognosis and postoperative fever $\geq 37^{\circ} \mathrm{C}$ duration in each stage of disease, statistically differences in OS between prolonged postoperative group and nonprolonged postoperative fever group were observed in both stage I $(P$ $=0.0046$, Fig. 4a) and stage II / III $(P=0.046$, Fig. 5a). Furthermore, statistically differences in DSS between prolonged postoperative group and nonprolonged postoperative fever group were also observed in both stage 


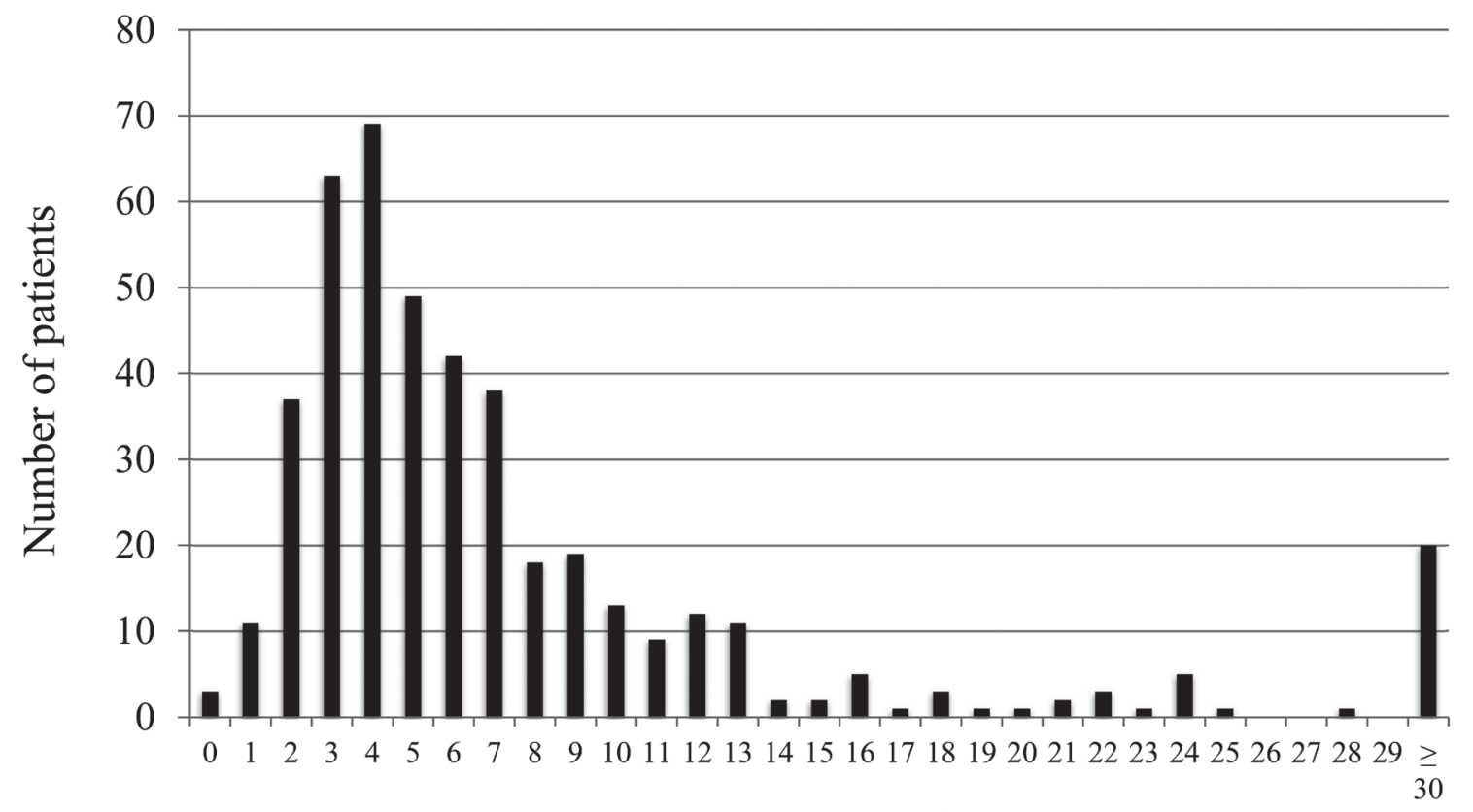

Postoperative fever $\geq 37^{\circ} \mathrm{C}$ duration

Fig. 2. Histogram of postoperative fever $\geq 37^{\circ} \mathrm{C}$ duration.

I $(P=0.0088$, Fig. 4b) and stage II / III $(P=0.023$, Fig. $5 b)$. Multivariate analysis indicated that postoperative fever $\geq 37^{\circ} \mathrm{C}$ duration was an independent prognostic indicator (Table 2).

Among the 442 patients included in this study, 85 patients (19.2\%) developed Clavien-Dindo classification infectious complications grade $\geq 2$. Five-year OS was $69.0 \%$ vs. $78.3 \%$ in patients with infectious complications grade $\geq 2$ vs. those without infectious complications grade $\geq 2$, respectively; the difference was statistically significant $(P=0.029)$. Postoperative fever duration was $20.5 \pm 27.5$ days vs. $5.9 \pm 4.8$ days in patients with infectious complications grade $\geq 2$ vs. those without infectious complications grade $\geq 2$, respectively; the difference was statistically significant $(P<0.0001$, Fig. 6).

Because it is likely that the presence of postoperative complications worsened prognosis in the prolonged postoperative fever group, we then determined the prognostic significance of postoperative fever in patients without postoperative infectious complications. Fiveyear OS was $69.7 \%$ vs. $84.0 \%$ in patients with prolonged fever vs. those without prolonged fever, respectively, and the difference was statistically significant $(P=0.0067$, Fig. 7a). Five-year DSS was $85.9 \%$ vs. $93.1 \%$ in patients with prolonged fever vs. those without prolonged fever, respectively, and the difference was statistically significant $(P=0.041$, Fig. $7 b)$.

\section{DISCUSSION}

It is widely accepted that inflammation plays a role in tumor pathogenesis, and that an inflammatory microenvironment is an essential component of all tumors. ${ }^{4}$ A recent study also demonstrated that postoperative inflammation worsened the prognosis of cancer patients. ${ }^{5}$ In routine clinical settings, some serum markers, such as C-reactive protein (CRP) and white blood cell, neutrophil, and thrombocyte counts, are used to evaluate patients' inflammation status. Other indicators, including NLR and PLR have also been developed to evaluate inflammation status. Postoperative values of these serum markers and indicators are closely associated with prognosis in cancer patients. ${ }^{5,6}$ We also previously reported that postoperative neutrophil-lymphocyte ratio measured 1 month after operation was significantly correlated with prognosis in gastric cancer patients. ${ }^{7}$

Fever is a simple and useful indicator to evaluate patients' inflammation status and is easier and more noninvasive than measuring serum markers. However, the prognostic significance of fever remains unclear in gastric cancer patients. Regarding the correlation between prognosis and fever, Yan et al. demonstrated that postoperative fever was an independent prognostic factor for relapse-free survival in node-negative breast cancer patients. ${ }^{8}$ They defined fever as an oral temperature $\geq 38^{\circ} \mathrm{C}$ in one week postoperation, which was similar to other reports. ${ }^{9}$ Feng et al. reported that duration of 
Table 1. Relationships between the duration of postoperative fever ( $\geq 37$ degree) and clinicopathological variables in patients with gastric cancer

\begin{tabular}{|c|c|c|}
\hline Variable & Postoperative fever $\geq 37^{\circ} \mathrm{C}$ duration [mean $\pm \mathrm{SD}$ (days)] & $P$ value \\
\hline Age (years) & & 0.53 \\
\hline$<70(n=216)$ & $7.8 \pm 11.0$ & \\
\hline$\geq 70(n=226)$ & $9.5 \pm 16.3$ & \\
\hline Sex & & 0.95 \\
\hline Male $(n=326)$ & $9.3 \pm 15.8$ & \\
\hline Female $(n=116)$ & $7.1 \pm 6.1$ & \\
\hline Tumor size & & 0.11 \\
\hline$<4 \mathrm{~cm}(n=279)$ & $8.0 \pm 14.1$ & \\
\hline$\geq 4 \mathrm{~cm}(n=163)$ & $9.8 \pm 13.7$ & \\
\hline Histology & & 0.68 \\
\hline Differentiated $(n=241)$ & $8.2 \pm 11.9$ & \\
\hline Undifferentiated $(n=201)$ & $9.3 \pm 16.2$ & \\
\hline Depth of invasion & & 0.0072 \\
\hline $\mathrm{T} 1(n=282)$ & $7.9 \pm 14.0$ & \\
\hline $\mathrm{T} 2 / \mathrm{T} 3 / \mathrm{T} 4(n=160)$ & $10.0 \pm 13.8$ & \\
\hline Lymph node metastasis & & 0.081 \\
\hline Absent $(n=340)$ & $8.3 \pm 14.5$ & \\
\hline Present $(n=102)$ & $9.8 \pm 12.1$ & \\
\hline Lymphatic involvement & & 0.086 \\
\hline Absent $(n=184)$ & $7.1 \pm 7.4$ & \\
\hline Present $(n=258)$ & $9.8 \pm 17.1$ & \\
\hline Venous involvement & & 0.025 \\
\hline Absent $(n=230)$ & $6.9 \pm 7.1$ & \\
\hline Present $(n=212)$ & $10.6 \pm 18.6$ & \\
\hline Stage of disease & & 0.073 \\
\hline $\mathrm{I}(n=308)$ & $8.1 \pm 13.8$ & \\
\hline II / III $(n=134)$ & $10.0 \pm 14.5$ & \\
\hline Approach & & 0.027 \\
\hline Open $(n=254)$ & $9.2 \pm 15.2$ & \\
\hline Laparoscopy $(n=188)$ & $8.1 \pm 15.8$ & \\
\hline Lymph node dissection a & & 0.69 \\
\hline $\mathrm{D} 0 / 1(n=303)$ & $9.0 \pm 15.7$ & \\
\hline D2 and more $(n=139)$ & $8.1 \pm 9.1$ & \\
\hline Gastrectomy & & 0.0015 \\
\hline Total $(n=95)$ & $11.4 \pm 17.0$ & \\
\hline Proximal and distal $(n=347)$ & $7.9 \pm 13.0$ & \\
\hline
\end{tabular}

${ }^{\text {a }}$ Lymph node dissection: D0-D2, extent of lymph node dissection.

fever $\geq 38^{\circ} \mathrm{C}$ for over $48 \mathrm{~h}$, but not less than $48 \mathrm{~h}$, was significantly associated with poorer prognosis in gastric cancer patients, ${ }^{10}$ indicating that fever duration might be also important when considering the correlation between fever and prognosis. In our study, we first determined the prognostic significance of postoperative fever duration and maximum postoperative temperature. ROC analysis for OS indicated that the AUC of 
(a)

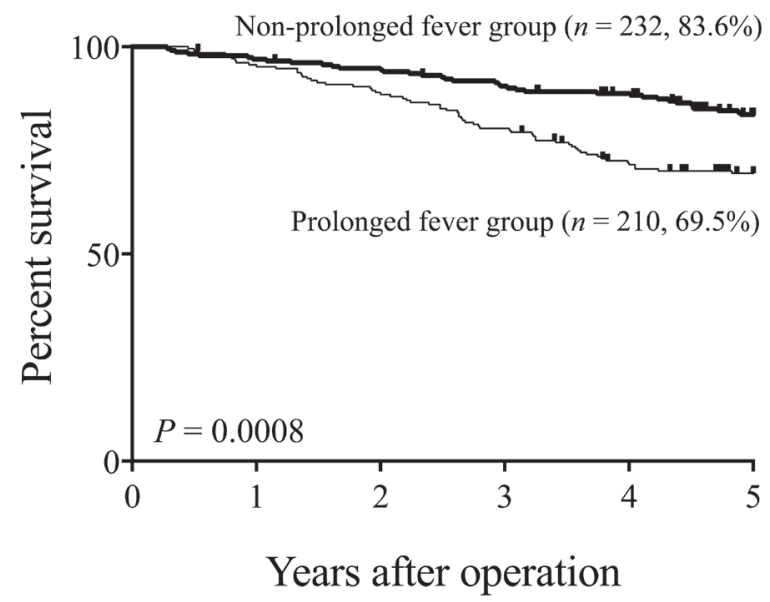

(b)

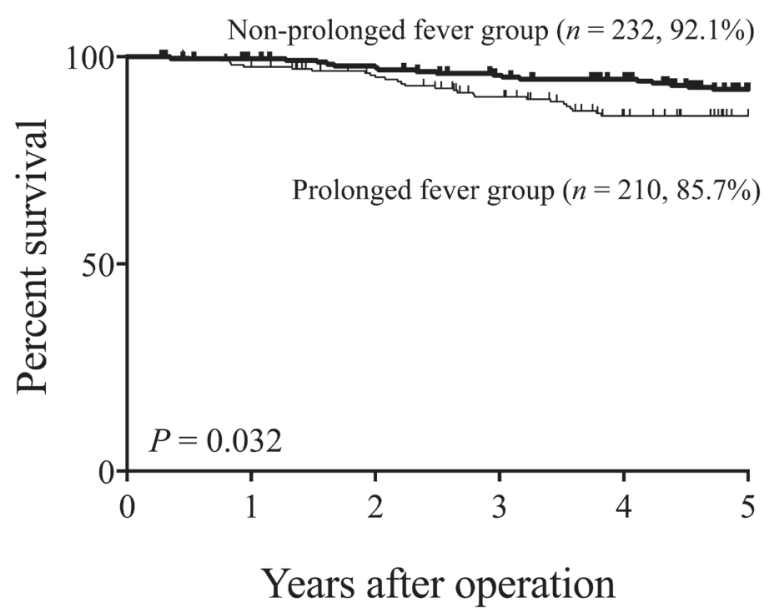

Fig. 3. (a) Overall survival curves based on postoperative fever $\geq 37^{\circ} \mathrm{C}$ duration. (b) Disease specific survival curves based on postoperative fever $\geq 37^{\circ} \mathrm{C}$ duration.

(a)

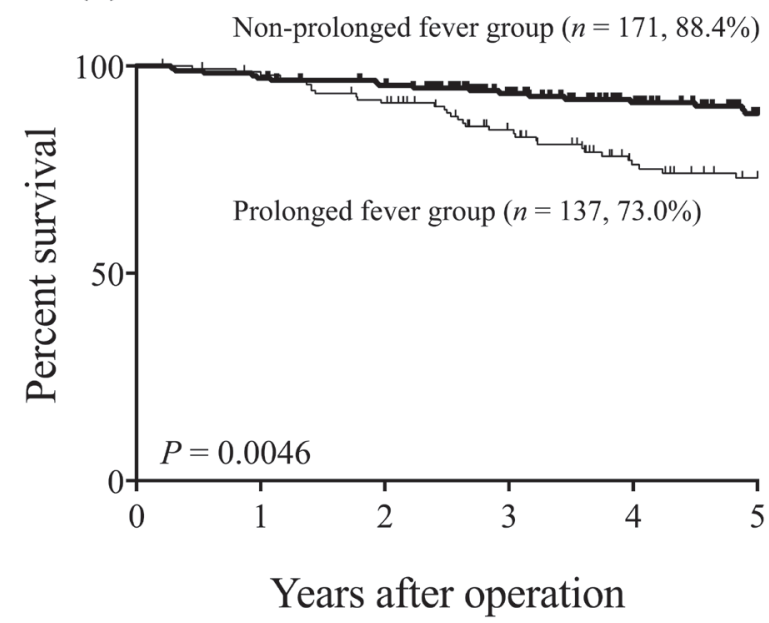

(b)

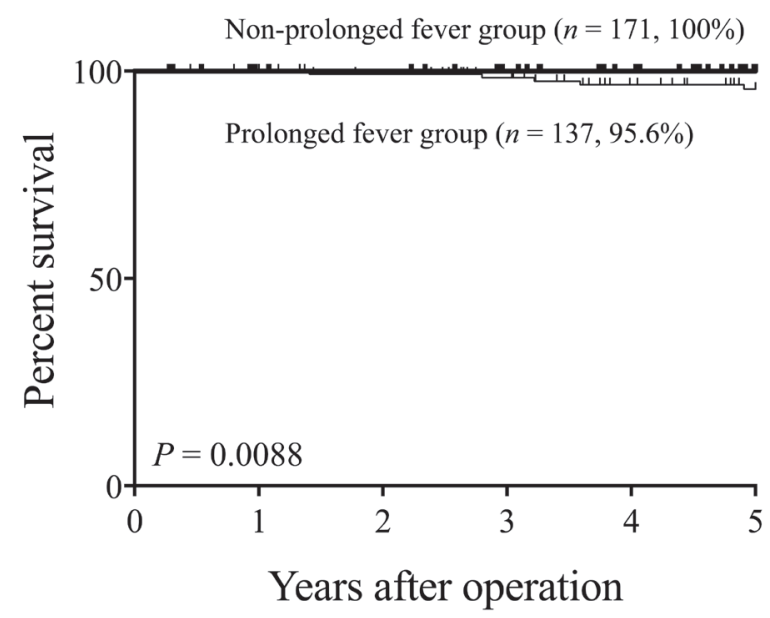

Fig. 4. Survival curves based on postoperative fever $\geq 37^{\circ} \mathrm{C}$ duration in stage I patients. (a) Overall survival curves, (b) Disease specific survival curves.

Table 2. Multivariate analyses of factors prognostic of overall survival in patients with gastric cancer

\begin{tabular}{|c|c|c|c|}
\hline Variable & $P$ value & HR & $95 \% \mathrm{CI}$ \\
\hline Age (years) ${ }^{a}$ & $<0.0001$ & 1.063 & $1.043-1.084$ \\
\hline Tumor size $(\mathrm{cm})^{\mathrm{a}}$ & 0.0012 & 1.119 & $1.046-1.197$ \\
\hline Lymph node metastasis $(\mathrm{N} 0-\mathrm{N} 3)^{b}$ & 0.021 & 1.28 & $1.037-1.579$ \\
\hline Lymphatic involvement $(\mathrm{Ly} 0-3)^{c}$ & 0.0047 & 1.424 & $1.114-1.821$ \\
\hline Duration of postoperative fever $(\geq 6 \mathrm{vs} .<6$ ) & 0.0031 & 1.745 & $1.206-2.525$ \\
\hline
\end{tabular}

${ }^{a}$ Continuous variable. ${ }^{b}$ Lymph node metastasis: N0, no regional lymph node metastasis; N1, metastasis in 1-2 regional lymph nodes; N2, metastasis in 3-6 regional lymph nodes; N3, metastasis in 7 or more regional lymph nodes. ${ }^{\mathrm{c}}$ Lymphatic involvement: Ly0-Ly3, grade of lymphatic invasion. CI, confidence interval; HR, hazard ratio. 
(a)

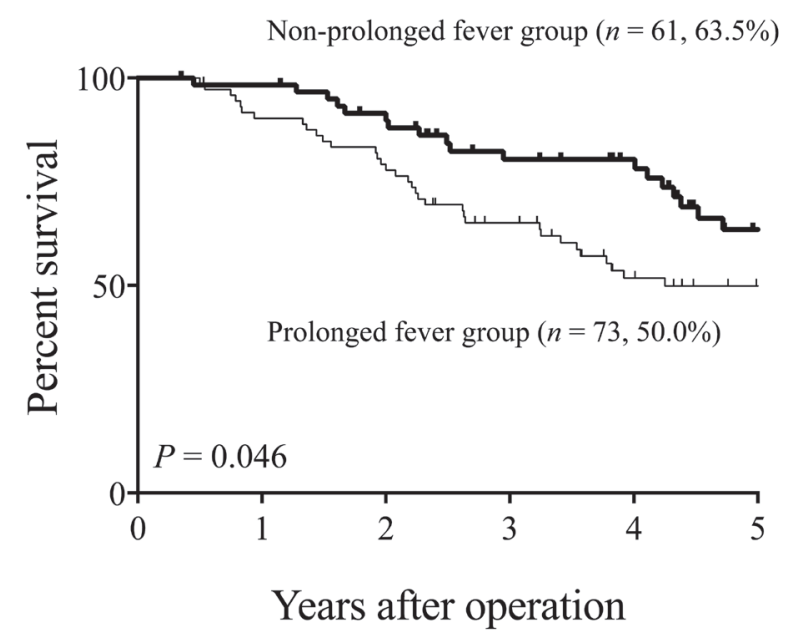

(b)

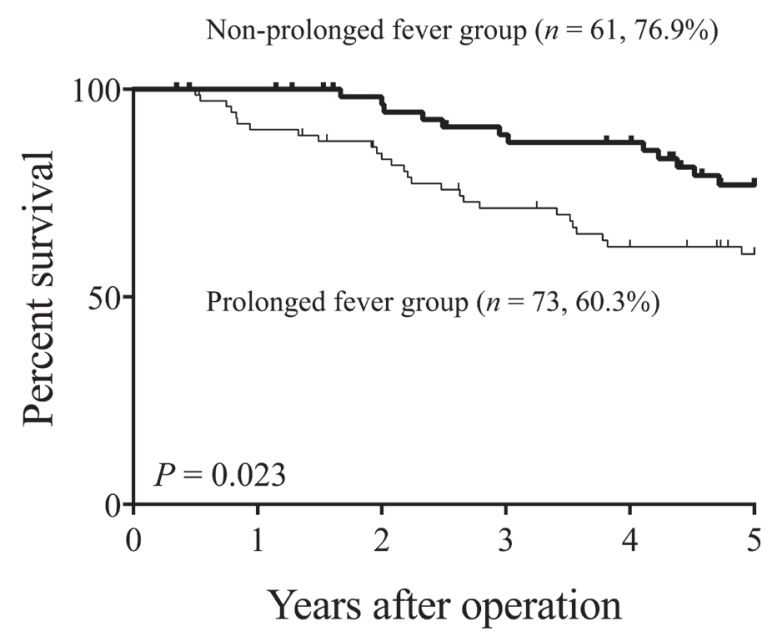

Fig. 5. Survival curves based on postoperative fever $\geq 37^{\circ} \mathrm{C}$ duration in stage II / III patients. (a) Overall survival curves, (b) Disease specific survival curves.

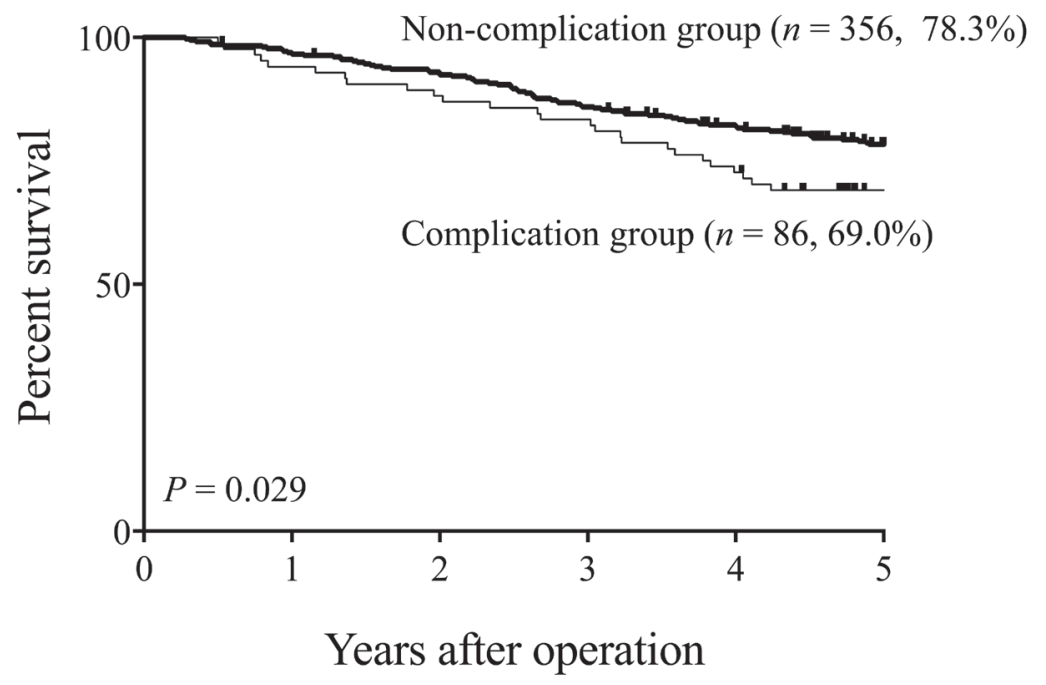

Fig. 6. Overall survival curves based on the presence of Clavien-Dindo classification postoperative infectious complications grade $\geq 2$.

postoperative fever $\left(\geq 37^{\circ} \mathrm{C}\right)$ duration was 0.6168 , which was higher than that of postoperative fever $\geq 37.5^{\circ} \mathrm{C}$ duration, postoperative fever $\geq 38.0^{\circ} \mathrm{C}$ duration, or maximum postoperative temperature, indicating that postoperative fever $\geq 37^{\circ} \mathrm{C}$ duration was more useful than the other indicators to predict prognosis in gastric cancer patients. With regard to the correlation between postoperative fever $\geq 37^{\circ} \mathrm{C}$ duration and clinicopathological characteristics, postoperative fever $\geq 37^{\circ} \mathrm{C}$ duration was significantly longer in patients with advanced gastric cancer than those with early gastric cancer. This can be explained by the extension of gastrectomy. Our data indicated that postoperative fever $\geq 37^{\circ} \mathrm{C}$ duration was significantly longer in patients who underwent total gastrectomy than in those who underwent either distal or proximal gastrectomy. In fact, total gastrectomy was performed more frequently in patients with advanced GC than in those with early GC in this study $(33.8 \%$ vs. $14.5 \%)$.

Five-year OS rates were statistically significantly different between the prolonged- and nonprolonged postoperative fever groups. Statistically differences in OS between prolonged postoperative group and nonprolonged postoperative fever group were also 
(a)

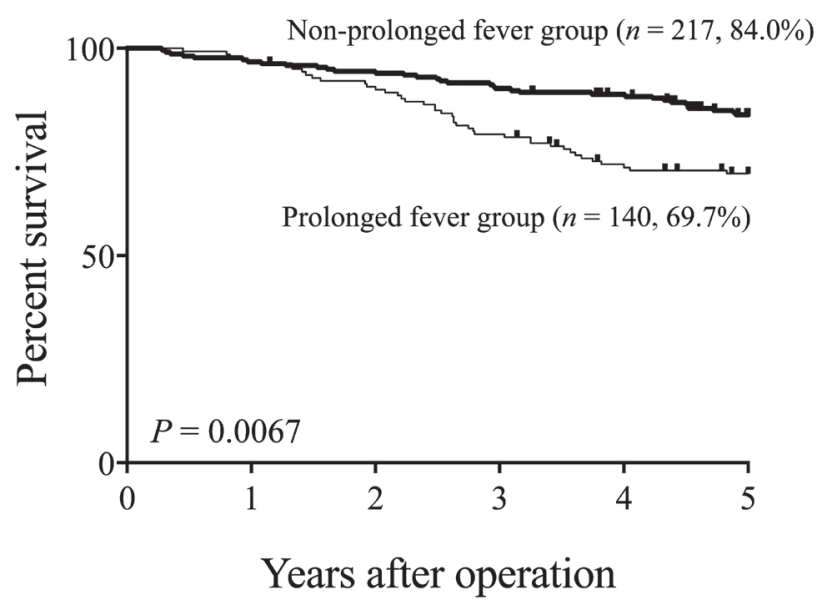

(b)

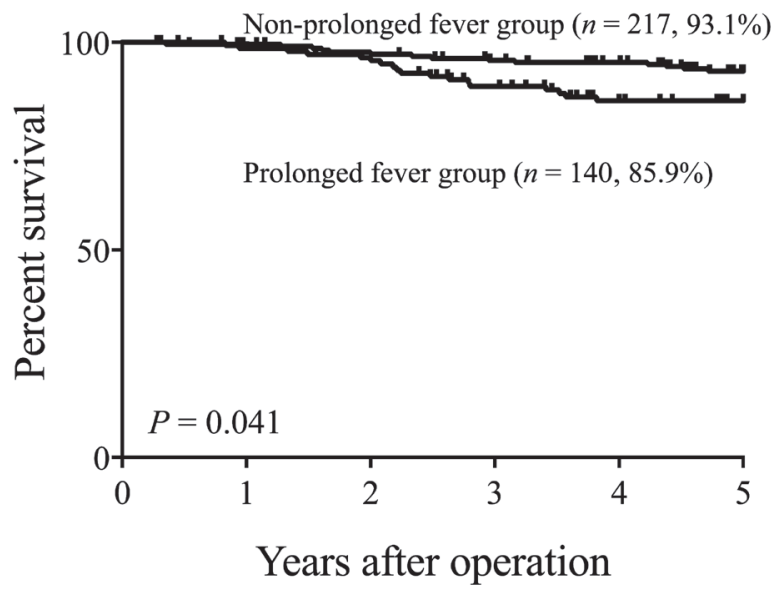

Fig. 7. (a) Overall survival curves based on postoperative fever $\geq 37^{\circ} \mathrm{C}$ duration in patients without Clavien-Dindo classification postoperative infectious complications grade $\geq 2$. (b) Disease-specific survival based on postoperative fever $\geq 37^{\circ} \mathrm{C}$ in patients without Clavien-Dindo classification postoperative infectious complications grade $\geq 2$.

observed in both stage I and stage II / III, indicating that postoperative fever $\geq 37^{\circ} \mathrm{C}$ duration was a useful prognostic indicator regardless of stage of disease in GC patients. In fact, multivariate analysis indicated that postoperative fever $\geq 37^{\circ} \mathrm{C}$ duration was an independent prognostic indicator. Of importance is that NLR and PLR, which were though to reflect patient's inflammation status and were reported to be prognostic indicators in GC patients, were not independent prognostic indicators in the current study, emphasizing the usefulness of postoperative fever $\geq 37^{\circ} \mathrm{C}$ duration as a prognostic indicator in GC patients.

Postoperative fever $\geq 37^{\circ} \mathrm{C}$ duration was used for survival analysis in the current study. This does not mean that $37^{\circ} \mathrm{C}$, which is close to normal body temperature, has prognostic impact. In this regard, postoperative fever $\geq 38^{\circ} \mathrm{C}$ durations was significantly longer in prolonged postoperative fever group than in nonprolonged postoperative fever group. Furthermore, maximum postoperative temperature was significantly higher in prolonged postoperative fever group than in nonprolonged postoperative fever group. Therefore, we think that postoperative fever $\geq 37^{\circ} \mathrm{C}$ duration is one of the best indicators to reflect the extent of patient's inflammation status.

Postoperative infectious complications induce prolonged postoperative inflammation. A close correlation between postoperative infectious complications and poor prognosis has been reported in extrahepatic biliary malignancies, ${ }^{11}$ hepatocellular carcinoma, ${ }^{12}$ esophageal cancer, ${ }^{13}$ colorectal cancer, ${ }^{14}$ and gastric cancer. ${ }^{15}$ Similarly, the prognosis of gastric cancer patients with postoperative infectious complications was significantly worse than for those without postoperative infectious complications in our study. Our results also demonstrated that postoperative fever $\geq 37^{\circ} \mathrm{C}$ duration was significantly longer in those with postoperative infectious complications compared with those without postoperative infectious complications. Therefore, it is likely that the poor prognosis seen in patients with prolonged postoperative fever might be secondary to the presence of postoperative infectious complications. However, this was not supported in our study because prolonged postoperative fever was significantly correlated with poor prognosis even in patients without postoperative infectious complications. Saito et al. also reported that CRP elevation is a more reliable indicator of survival after gastric cancer surgery than postoperative complication occurrence. ${ }^{6}$

The detailed mechanisms of the influence of postoperative fever on prognosis in cancer patients are still poorly understood. Cytokines appear to play important roles and have been implicated as pivotal mediators of the pyrogenic or antipyretic process, including proinflammatory tumor necrosis factor-alpha, and interleukin- 6 and- 1 as well as the anti- inflammatory interleukin-1 receptor antagonist and interleukin-10. ${ }^{16}$ The levels of cytokines with opposite function determine the occurrence and magnitude of fever. Surgical and anesthetic stress may elicit an imbalance between pro- and anti-inflammatory cytokines leading to fever. ${ }^{16}$ Several reports demonstrated a stimulatory role for 
tumor necrosis factor-alpha, and interleukin-1 and -6 in the proliferation of breast tumors. ${ }^{17}$ Therefore, elevated expressions of these proinflammatory cytokines might cooperate to exacerbate pre-existing subclinical lesions and promote recurrence and death in patients with postoperative fever. This may explain the detrimental effect of fever on prognosis in our study. Further investigations regarding the detailed mechanisms of how postoperative fever affects the prognosis of gastric cancer patients are urgently required.

We have demonstrated that postoperative fever duration is useful in predicting the disease specific prognosis of stage I GC patients in this study. Therefore, the postoperative fever duration can be used to identify the patients with high possibility of recurrence who might need intensive follow-up for early detection of recurrence. The Japanese Gastric Cancer Treatment Guideline recommends adjuvant chemotherapy with S-1 for a year in stage II/III GC patients who underwent gastrectomy with D2 lymph node dissection based on the results of the Adjuvant Chemotherapy Trial of TS-1 for Gastric Cancer (ACTS-GC). ${ }^{18}$ Since the postoperative fever duration was also useful in predicting the disease specific prognosis of stage II / III GC patients who were candidates of adjuvant chemotherapy, it can be also used to identify the patients with high possibility of recurrence who might need more intensive adjuvant chemotherapy to prevent recurrence.

NSAIDs or acetaminophen can lower the body temperature. Because there was a close correlation between prolonged postoperative fever and poor prognosis in the current study, it might be possible that prophylactic usage of those drugs improve the prognosis of GC patients. Furthermore, it might be important for surgeons to try to minimize the postoperative inflammatory response to improve prognosis. In this regard, laparoscopic surgery might be one of the ways to minimize the postoperative inflammatory response because postoperative fever $\geq 37^{\circ} \mathrm{C}$ duration in patients who underwent laparoscopic surgery was significantly shorter than that in those who underwent open surgery in this study. These ideas must be confirmed in a prospective randomized controlled trial.

Our study has certain limitations. First, because it was retrospective, it was subject to bias. Second, we used 6 days as a cut-off value for postoperative fever $\geq$ $37^{\circ} \mathrm{C}$ based on the ROC analysis; however, the optimal cut-off value for postoperative fever duration remains unclear. Third, the number of patients included in our study was small, and the results must be confirmed in a large-scale, prospective, randomized, controlled trial.

In conclusion, our study suggested the potential of prolonged postoperative fever to predict prognosis in gastric cancer patients. Because postoperative fever can be measured quickly, easily, and noninvasively, it may be a useful prognostic marker in routine clinical settings.

Acknowledgments: We thank Jane Charbonneau, DVM, from Edanz Group (www.edanzediting.com/ac) for editing a draft of this manuscript.

\section{The authors declare no conflict of interest.}

\section{REFERENCES}

1 Bray F, Ferlay J, Soerjomataram I, Siegel RL, Torre LA, Jemal A. Global cancer statistics 2018: GLOBOCAN estimates of incidence and mortality worldwide for 36 cancers in 185 countries. CA Cancer J Clin. 2018;68:394-424. DOI: 10.3322/caac.21492, PMID: 30207593

2 Fujiya K, Tokunaga M, Makuuchi R, Nishiwaki N, Omori H, Takagi W, et al. Early detection of nonperitoneal recurrence may contribute to survival benefit after curative gastrectomy for gastric cancer. Gastric Cancer. 2017;20(suppl 1):141-9. DOI: 10.1007/s10120-016-0661-x, PMID: 27778124

3 Japanese Gastric Cancer Association. Japanese classification of gastric carcinoma: 3rd English edition. Gastric Cancer. 2011;14:101-12. DOI: 10.1007/s10120-011-0041-5, PMID: 21573743

4 Grivennikov SI, Greten FR, Karin M. Immunity, inflammation, and cancer. Cell. 2010;140:883-99. DOI: 10.1016/ j.cell.2010.01.025, PMID: 20303878

5 Okumura Y, Hiki N, Kumagai K, Ida S, Nunobe S, Ohashi $\mathrm{M}$, et al. Postoperative prolonged inflammatory response as a poor prognostic factor after curative resection for gastric cancer. World J Surg. 2017;41:2611-8. DOI: 10.1007/s00268017-4032-5, PMID: 28451762

6 Saito T, Kurokawa Y, Miyazaki Y, Makino T, Takahashi $\mathrm{T}$, Yamasaki $\mathrm{M}$, et al. Which is a more reliable indicator of survival after gastric cancer surgery: postoperative complication occurrence or C-reactive protein elevation? J Surg Oncol. 2015;112:894-9. DOI: 10.1002/jso.24067, PMID: 26458724

7 Miyatani K, Saito H, Kono Y, Murakami Y, Kuroda H, Matsunaga T, et al. Combined analysis of the pre- and postoperative neutrophil-lymphocyte ratio predicts the outcomes of patients with gastric cancer. Surg Today. 2018;48:300-7. DOI: 10.1007/s00595-017-1587-6, PMID: 28916967

8 Yan T, Yin W, Zhou L, Jiang Y, Shen Z, Shao Z, et al. Postoperative fever: the potential relationship with prognosis in node negative breast cancer patients. PLoS One. 2010;5:e15903. DOI: 10.1371/journal.pone.0015903, PMID: 21209958

9 Mitchell JD, Grocott HP, Phillips-Bute B, Mathew JP, Newman MF, Bar-Yosef S. Cytokine secretion after cardiac surgery and its relationship to postoperative fever. Cytokine. 2007;38:37-42. DOI: 10.1016/j.cyto.2007.04.009, PMID: 17572096

10 Feng F, Tian Y, Yang X, Sun L, Hong L, Yang J, et al. Postoperative fever predicts poor prognosis of gastric cancer. Oncotarget. 2017;8:62622-9. DOI: 10.18632/oncotarget.15979, PMID: 28977974 
11 Buettner S, Ethun CG, Poultsides G, Tran T, Idrees K, Isom CA, et al. Surgical site infection is associated with tumor recurrence in patients with extrahepatic biliary malignancies. J Gastrointest Surg. 2017;21:1813-20. DOI: 10.1007/s11605017-3571-2, PMID: 28913712

12 Margonis GA, Sasaki K, Andreatos N, Nishioka Y, Sugawara T, Amini N, et al. Prognostic impact of complications after resection of early stage hepatocellular carcinoma. J Surg Oncol. 2017;115:791-804. DOI: 10.1002/jso.24576, PMID: 28205284

13 Kataoka K, Takeuchi H, Mizusawa J, Igaki H, Ozawa S, Abe T, et al. Prognostic impact of postoperative morbidity after esophagectomy for esophageal cancer: exploratory analysis of JCOG9907. Ann Surg. 2017;265:1152-7. DOI: 10.1097/ SLA.0000000000001828, PMID: 27280509

14 Artinyan A, Orcutt ST, Anaya DA, Richardson P, Chen GJ, Berger DH. Infectious postoperative complications decrease long-term survival in patients undergoing curative surgery for colorectal cancer: a study of 12,075 patients. Ann Surg. 2015;261:497-505. DOI: 10.1097/SLA.0000000000000854, PMID: 25185465
15 Tokunaga M, Tanizawa Y, Bando E, Kawamura T, Terashima M. Poor survival rate in patients with postoperative intraabdominal infectious complications following curative gastrectomy for gastric cancer. Ann Surg Oncol. 2013;20:157583. DOI: 10.1245/s10434-012-2720-9, PMID: 23076557

16 Conti B, Tabarean I, Andrei C, Bartfai T. Cytokines and fever. Front Biosci. 2004;9:1433-49. DOI: 10.2741/1341, PMID: 14977558

17 Jiang S, Zhang HW, Lu MH, He XH, Li Y, Gu H, et al. MicroRNA-155 functions as an OncomiR in breast cancer by targeting the suppressor of cytokine signaling 1 gene. Cancer Res. 2010;70:3119-27. DOI: 10.1158/0008-5472.CAN-094250, PMID: 20354188

18 Japanese Gastric Cancer Association. Japanese gastric cancer treatment guidelines 2014 (ver. 4). Gastric Cancer. 2017;20:119. DOI: 10.1007/s10120-016-0622-4, PMID: 27342689 